Vulnerabilidades urbanas en los países andinos

(Bolivia, Ecuador, Perú)

\title{
Jean-Pierre Soulas (1947-2009)
}

\section{(2) OpenEdition}

Journals

Edición electrónica

URL: http://journals.openedition.org/bifea/2587

DOI: $10.4000 /$ bifea. 2587

ISSN: 2076-5827

\section{Editor}

Institut Français d'Études Andines

\section{Edición impresa}

Fecha de publicación: 1 diciembre 2009

Paginación: 973-976

ISSN: 0303-7495

\section{Referencia electrónica}

" Jean-Pierre Soulas (1947-2009) », Bulletin de l'Institut français d'études andines [En línea], 38 (3) | 2009 Publicado el 01 junio 2010, consultado el 18 noviembre 2020. URL : http://journals.openedition.org/ bifea/2587 ; DOI : https://doi.org/10.4000/bifea.2587

\section{c) (i)}

Les contenus du Bulletin de l'Institut français d'études andines sont mis à disposition selon les termes de la licence Creative Commons Attribution - Pas d'Utilisation Commerciale - Pas de Modification 4.0 International. 


\title{
In memoriam
}

\author{
JEAN-PIERRE SOULAS
}

(1947-2009)

Jean-Pierre Soulas, geólogo y ex investigador del IFEA, falleció el 31 de agosto de 2009. Después de su tesis de maestría en geología en Burdeos, realizada entre 1966 y 1970, escogió la ciudad de Besançon para preparar, entre 1970 y 1974, una tesis de doctorado que trató de la microtectónica de las montañas del Jura. En 1974, los Andes representaron para él un nuevo y rico campo de investigación en un ambiente marcado por manifestaciones de una tectónica reciente y actual. Se podrán apreciar sus resultados más relevantes más abajo en el listado (con seguridad incompleto) de sus publicaciones. Después de terminar su estadía como voluntario del servicio militar en el extranjero (VSNA, 1974-1976), JeanPierre Soulas trabajó en el IFEA como investigador (post-doctoral o pensionnaire) durante el periodo 1976-1978. Huelga decir el provecho científico que sacó de estos años en el Perú.

En 1979, viajó a Venezuela y Caracas para desempeñarse como consejero científico de la Fundación Venezolana de Investigaciones Sismológicas (FUNVISIS). Allí permaneció ocho años, a la cabeza de la dirección científica y técnica del departamento de Ciencias de la Tierra de la Fundación, donde se encargó de dirigir unas tesis y se dedicó a salidas de campo. Dictó también clases internacionales en los países andinos y El Caribe. Igual que en el Perú, sus investigaciones abarcaron la tectónica cuaternaria, las fallas activas, la sismo-tectónica y los riesgos sísmicos tan apremiantes para el conjunto de estos países.

En 1987, Jean-Pierre Soulas regresó a Francia y a Arcachon donde se instaló definitivamente y trabajó como consultor independiente en cartografía, en geología de campo, en geología aplicada a la ingeniería, en el estudio de las deformaciones tectónicas, de la geomorfología de las fallas activas y del riesgo tectónico. Efectuó varias misiones para Naciones Unidas en América Latina, en Turquía y en Chipre. 


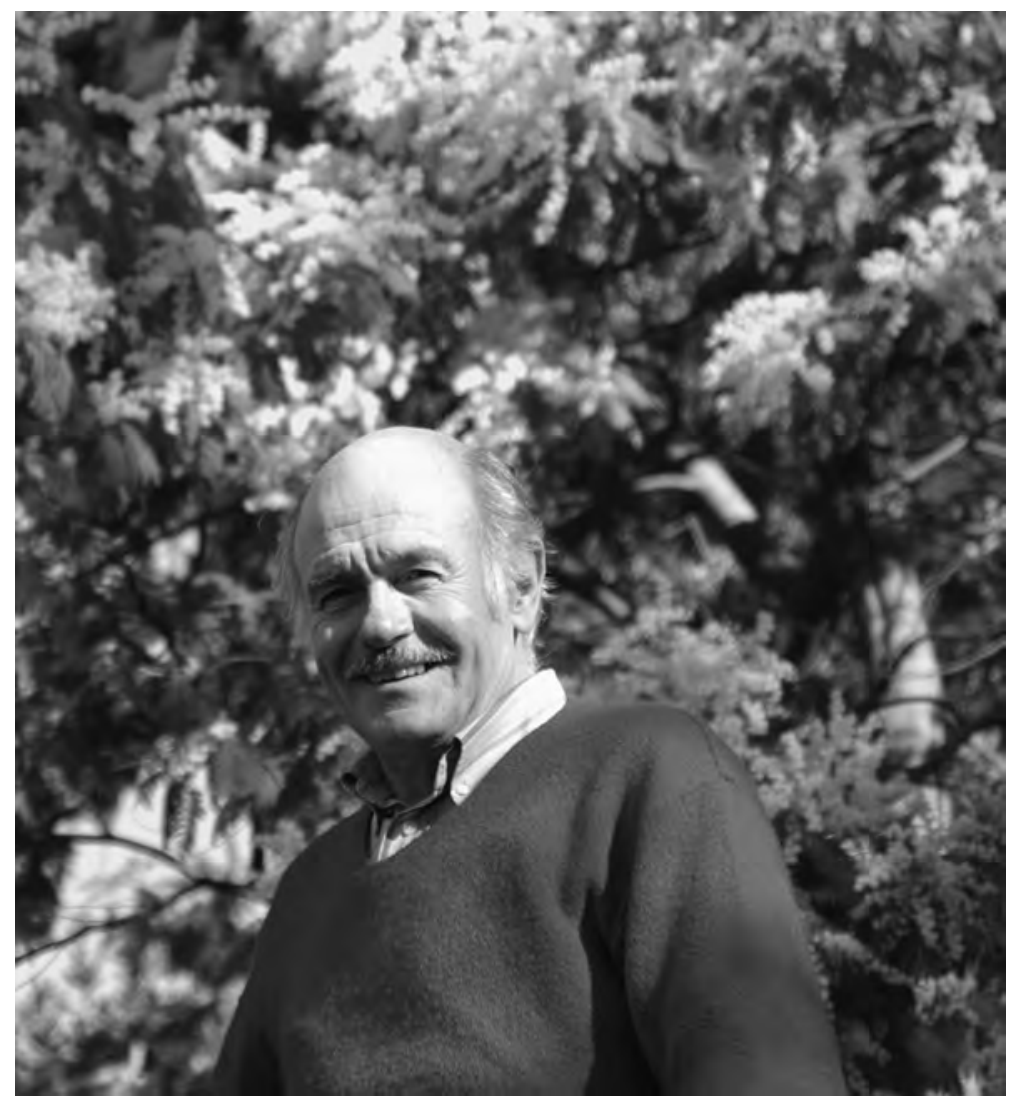

Fui a recoger a Jean-Pierre Soulas a su llegada al aeropuerto de Lima durante la primavera de 1974. Uno de los programas del IFEA vertía sobre el desarrollo de análisis tectónicos detallados en los Andes peruanos. En aquel entonces esta zona gozaba de un mejor conocimiento científico gracias, entre otros, a los trabajos François Mégard, Bernard Dalmayrac y René Marocco. Reemplazaba a Michel Ribeyrolles, joven geólogo fallecido en un accidente, y demostró de inmediato su deseo de irse a la sierra. Aficionado al riesgo, Jean-Pierre Soulas era huraño, curioso y discreto, muy trabajador y exigente consigo mismo y con los demás, amateur en estos difíciles terrenos andinos a los que iba a dedicar, junto con numerosos colegas suyos, sus conocimientos de geólogo en tectónica. No descuidaba ningún detalle en sus análisis y dudaba sistemáticamente de sus propios resultados, a tal punto que se le tildó de maniático. Nunca se dejó influenciar por alguna hipótesis de moda.

Su trayectoria de investigador-consultor, después de haber ocupado un puesto en el IFEA, no fue convencional; pues también respondió a la apertura, disponibilidad e interés que sabía demostrar Jean-Pierre Soulas para atender pedidos no académicos que emanaban de la sociedad civil, para utilizar un término actual. Recuerdo 
haberlo acompañado a la Defensa Civil peruana, después de un importante deslizamiento en el valle del Mantaro en mayo de 1974, y haber compartido con él otras actividades para diversas entidades mineras. Se entiende mejor así su compromiso como ciudadano y como miembro del consejo municipal de su municipalidad de Arcachon.

\section{Bibliographie}

LAVENU, A. \& SOULAS, J.-P., 1974 - Observations de microfailles plio-quaternaires en distension le long de la Côte Sud du Pérou. Bulletin de I'Institut Français d'Études Andines, 3 (2): 39-48.

Resumen (extracto): Le long de la frange littorale, entre Paracas et Camaná, nous avons observé des microfailles en extension, affectant des terrains quaternaires ou plio-quaternaires. Une étude statistique, sur diagrammes, de ces failles montre qu'il existe deux zones où les directions des diverses familles sont différentes

SOULAS, J.-P., 1975 - La chaîne andine du Pérou Central: chronologie, orientation et styles des phases tectoniques du Tertiaire Supérieur - Aperçus sur la Tectonique Quaternaire. Bulletin de I'Institut Français d'Études Andines, 4 (3-4): 127-156.

Resumen (extracto): Les phases tectoniques mio-pliocènes sont étudiées dans plusieurs secteurs des Andes Centrales péruviennes. Au sud de Huancavelica (Choclococha, Huachocolpa, Lircay) on note une phase de déformation cassante pliocène, selon une direction de raccourcissement $\mathrm{N} 090$ (entre 8 et 4,5 M.A.).

SOULAS, J.-P., 1975 - Las fases tectónicas jóvenes de los Andes centrales del Perú. Boletín de la Sociedad geológica del Perú, 50: 77-86; Lima.

SOULAS, J.-P., 1977 - Les phases tectoniques andines du Tertiaire supérieur, résultats d'une transversale Pisco-Ayacucho, Pérou central. Comptes rendus hebdomadaires des séances de l'académie des sciences. Série D, 84: 2207-2210.

SOULAS, J.-P., 1977 - Las fases tectónicas del Terciario Superior en Perú, corte Ayacucho Pisco. Boletín de la Sociedad geológica del Perú, 57-58: 59-72.

SOULAS, J.-P., 1978 - Tectonique Quaternaire: la côte pacifique et la chaîne andine du Pérou Central. Revue de géographie physique et de géologie dynamique, 20 (5): 399-414.

SOULAS, J.-P., SINGER, A. \& GALLARDO, C., 1980 - Les failles actives de la régión NordOrientale du Vénézuela: 392.

SOULAS, J.-P., 1981 - Recent changes in the quaternary stress field of the venezuelan Andes. EOS, 62 (45): 1026; San Francisco.

AGGARWAL, Y. P, SOULAS, J.-P. \& GARCÍA, D., 1983 - Contemporary tectonics of the venezuelan Andes and Northern Colombia. In: $10^{\circ}$ Caribbean geological conference: 16-17; Cartagena.

SOULAS, J.-P., 1983 - Mapa preliminar de Tectónica Cuaternaria de Venezuela. Acta científica venezolana, 34 (1): 513; Caracas.

SOULAS, J.-P., 1983 - Tectónica cuaternaria de la mitad Sur de los Andes venezolanos. Grandes rasgos. Acta científica venezolana, 34 (1): 525; Caracas. 
SOULAS, J.-P., SINGER, A. \& GIRALDO, C., 1983 - Tectónica cuaternaria de la región nororiental de Venezuela y de Trinidad. Grandes rasgos. Acta científica venezolana, 34 (1): 532.

BELTRÁN, C., SINGER, A. \& SOULAS, J.-P., 1983 - Discriminación entre evidencias diagnósticas y evidencias ficticias de fallamiento activo en ambiente tropical húmedo. Acta científica venezolana, 34 (1): 522.

SOULAS, J.-P., 1985 - Preliminary neotectonic map of South America. National report of Venezuela. In: Mapa neotectónico preliminar para América del Sur. Preliminary neotectonic map for South America (Programa para la mitigación de los efectos de los terremotos en la región andina [Proyecto SISRA]): 105-110; Lima: Centro regional de sismología para América del Sur, CERESIS.

SOULAS, J.-P., 1985 - Mapa neotectónico preliminar de América del Sur. Informe nacional de Venezuela. In: Mapa neotectónico preliminar para América del Sur. Preliminary neotectonic map for South America (Programa para la mitigación de los efectos de los terremotos en la región andina [Proyecto SISRA]): 51-63; Lima: Centro regional de sismología para América del Sur, CERESIS.

SOULAS, J.-P., 1986 - Neotectonics and active tectonics in Venezuela and neighboring regions. Bulletin INQUA, 9: 105.

SOULAS, J.-P., 1986 - Neotectonis of the western flank of the Venezuelan Andes betwen $70^{\circ} 30^{\prime} \mathrm{W}$ and $71^{\circ} 00^{\prime} \mathrm{W}$ : faults of Bocono, Valera, Tuñame, Piñango and Piedmont. Bulletin INQUA, 9: 106.

SOULAS, J.-P., SINGER, A. \& LUGO, M., 1987 - Proyecto SUMANDES. FUNVISIS-Maraven; Caracas.

SOULAS, J.-P., 1989 - Définition de I'actuelle limite Sud de la Plaque Caraïbe, 1 p.; Barcelona.

SOULAS, J.-P., 1992 - Segmentation et séismes caractéristiques. In: Frontiers in fundamental seismology: 14; Strasbourg: École et observatoire de physique du globe.

SOULAS, J.-P., s./f. - Observaciones sobre la tectónica del sector Choclococha - Huachocolpa - Lircay (Sur de Huancavelica - Perú), 9 pp.; Lima. 
Pedidos: IFEA, Casilla 18-1217, Lima 18 - Perú, Tel. 4476070

Fax: 4457650 - E-mail: postmaster@ifea.org.pe Web: http://www.ifeanet.org

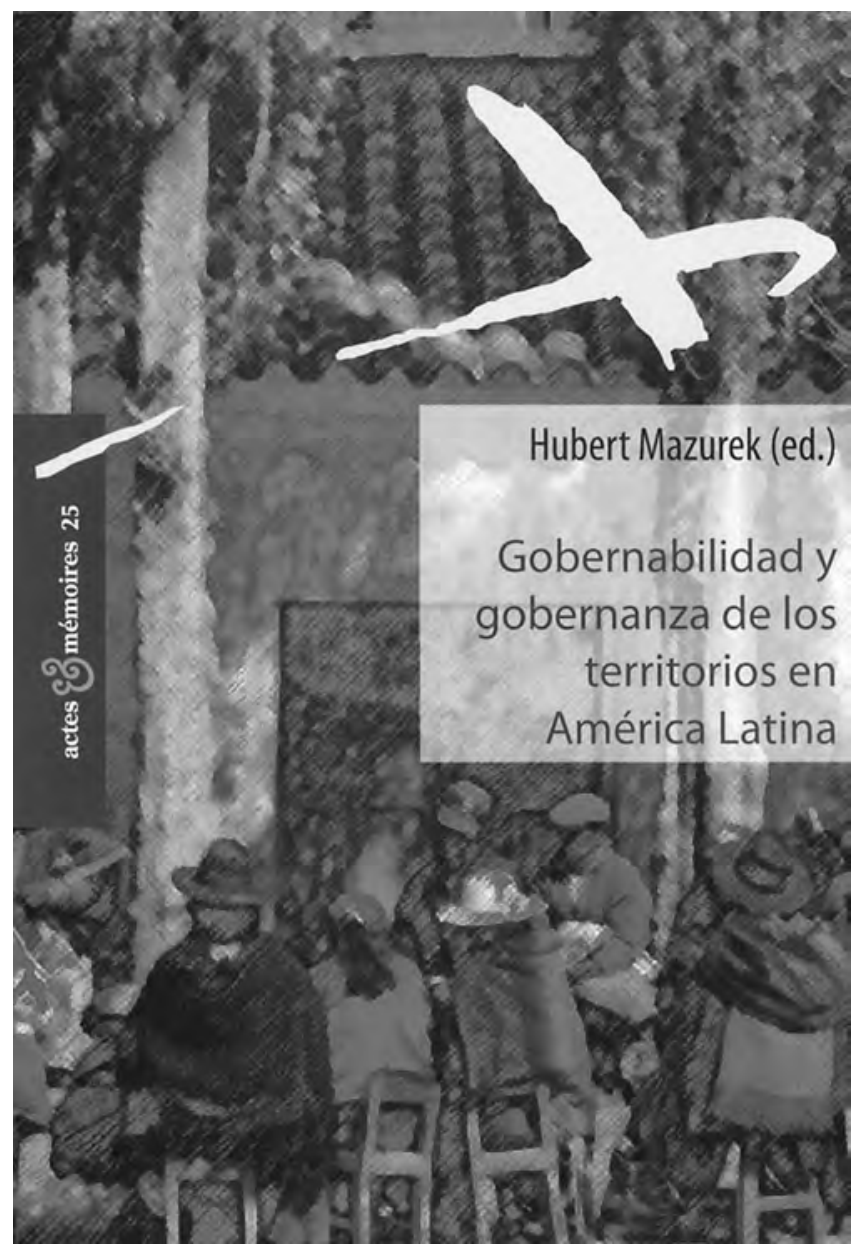

Coedición: Institut français d'études andines (IFEA, UMIFRE 17, CNRS-MAEE) - Institut de Recherche pour le Développement (IRD) - Cooperación Regional para los Países Andinos - Universidad Mayor de San Simón, Centro de Estudios Superiores Universitarios 

SE TERMINÓ DE IMPRIMIR EN LOS TALLERES GRÁFICOS DE Tarea Asociación Gráfica Educativa Pasaje María Auxiliadora 156-164 - Breña CoRreo E.: TAREAGRAFICA@ $@$ TAREAGRAFICA.COM TeléF. 332-3229 FAX: 424-1582 Marzo 2010 Lima - Perú 
